\title{
WEAK SEQUENTIAL COMPLETENESS IN SPACES OF OPERATORS ${ }^{1}$
}

\author{
J. M. BAKER
}

Throughout, $X$ and $Y$ will be nontrivial normed linear spaces over the field of complex numbers, $E$ the space $\mathscr{L}(X, Y)$ of continuous linear transformations from $X$ into $Y$ equipped with the usual norm, which we denote by $\mu, W$ a linear subspace of the dual $Y^{\prime}$ of $Y$, and $\omega$ the set of positive integers. The norm-closed unit ball in $X$, for example, is written $S_{X}$, and the canonical embedding $\phi$ from $Y$ into $W^{\prime}$ is defined by $\phi(y)(w)=w(y)$. Each $f$ belonging to the tensor product $X \otimes W$ determines a continuous linear functional on $E$ since if $\sum_{i=1}^{k} x_{i} \otimes w_{i}$ is any representation of $f$ then

$$
|f(T)|=\left|\sum_{i=1}^{k} w_{i}\left(T x_{i}\right)\right| \leqq \sum_{i=1}^{k}\left\|w_{i}\right\|\left\|x_{i}\right\|
$$

for each $T \in S_{E}$. It is easy to verify that the restriction to the subspace $X \otimes W$ of the dual norm in $E^{\prime}$ is a cross norm $\psi$ on $X \otimes W$. For each $W$, therefore, we let $F_{W}=X \otimes_{\psi} W$, where $\otimes_{\psi}$ denotes the tensor product under the cross norm $\psi$, and consider $F_{W}$ as a subspace of $E^{\prime}$. Also, we will consider $X^{\prime} \otimes_{\lambda} Y$ canonically embedded in $E$, where $\lambda$ is the least cross norm [6].

Following Dixmier [1], we define the characteristic of $W$ to be the number

$$
\nu(W)=\inf _{y \in \Gamma^{\prime} ; w \in S_{W}}|w(y)|, \quad\|y\|=1 .
$$

Thus, $0 \leqq \nu(W) \leqq 1$. Letting $W_{0}$ be the annihilator of $W$ in $Y$, it is evident that $W_{0}=\{0\}$ if $\nu(W)>0$ and that $W_{0}=\{0\}$ if and only if the topology $\sigma(Y, W)$ is Hausdorff.

In the work below, we are concerned with theorems dealing with relationships between the space $Y$ equipped with a topology $\sigma(Y, W)$ and the space $E$ equipped with the corresponding topology $\sigma\left(E, F_{W}\right)$. Let $\nu(W)>0$, and consider the family of seminorms $\left\{p_{x, w}\right\}$ defined by

$$
p_{x, w}(T)=|w(T x)| \quad(w \in W, x \in X)
$$

Presented to the Society, January 24, 1969 under the title Weak completeness in spaces of operators; received by the editors May 12, 1969 and, in revised form, October 17, 1969.

1 This work, which was supported in part by NSF Grant GP-7243, is a portion of the author's Florida State University dissertation written under the direction of Professor R. D. McWilliams. 
for each $T \in E$. For the case $W=Y^{\prime}$, this family of seminorms defines the weak operator topology [2] which is precisely $\sigma\left(E, F_{Y^{\prime}}\right)$. More generally, if $W$ is a subspace of $Y^{\prime}$ for which $\nu(W)>0$, the family of seminorms $\left\{p_{x, w}\right\}$ defines on $E$ a topology which is weaker than the weak operator topology and which is precisely $\sigma\left(E, F_{W}\right)$. Thus, operator theorists who are not interested in tensor products and crossnorms will find our work more transparent if they will view the results in a weak operator type setting.

Theorem. (i) If $\nu(W)=1$ and $Y$ is $\sigma(Y, W)$-sequentially complete, then $S_{E}$ is $\sigma\left(E, F_{W}\right)$-sequentially complete.

(ii) Let $\nu(W)>0$ and $W$ be norm-closed in $Y^{\prime}$. If $Y$ is $\sigma(Y, W)$-sequentially complete and $X$ is a Banach space, then $E$ is $\sigma\left(E, F_{W}\right)$-sequentially complete. Conversely, if each $\sigma\left(E, F_{W}\right)$-Cauchy sequence in $\left(X^{\prime} \otimes_{\lambda} Y\right) \cap S_{E}$ is $\sigma\left(E, F_{W}\right)$-convergent to some $T \in E$, then $Y$ is $\sigma(Y, W)$ sequentially complete.

(iii) Let $Y$ be a barrelled normed linear space. Suppose $Y^{\prime} \mid W$ is of countable Hamel dimension and $W$ is $\sigma\left(Y^{\prime}, Y\right)$-sequentially dense in $Y^{\prime}$. If $\nu(W)>0, Y$ is $\sigma(Y, W)$-sequentially complete, and $X$ is a Banach space, then $E$ is $\sigma\left(E, F_{W}\right)$-sequentially complete. Conversely, if every $\sigma\left(E, F_{W}\right)$-Cauchy sequence in $\left(X^{\prime} \otimes_{\lambda} Y\right) \cap S_{E}$ is $\sigma\left(E, F_{W}\right)$-convergent to an element $T \in E$, then $Y$ is $\sigma(Y, W)$-sequentially complete.

Proof. To prove (i) let $\left\{T_{n}\right\}$ be a $\sigma\left(E, F_{W}\right)$-Cauchy sequence in $S_{E}$. Then for each $x \in X$ the sequence $\left\{T_{n} x\right\}$ is $\sigma(Y, W)$-convergent in $Y$. We define a mapping $T$ from $X$ into $Y$ by writing $T x=y$ if and only if $y=\sigma(Y, W)-\lim T_{n} x$. Obviously, $T$ is linear. Since $\nu(W)=1$,

$$
\|T x\|=\sup _{w \in S_{W}} \lim _{n}\left|w\left(T_{n} x\right)\right| \leqq\|x\| .
$$

Thus, $T \in S_{E}$. Finally,

$$
\begin{aligned}
\lim _{n}\left(\sum_{i} x_{i} \otimes w_{i}\right)\left(T_{n}\right) & =\lim _{n} \sum_{i} w_{i}\left(T_{n} x_{i}\right) \\
& =\sum_{i} w_{i}\left(T x_{i}\right) \\
& =\left(\sum_{i} x_{i} \otimes w_{i}\right)
\end{aligned}
$$

for each $\sum_{i} x_{i} \otimes w_{i} \in F_{W}$.

We proceed to prove the first assertion of (ii) by considering the seminorm $p_{w}$ on $Y$ defined by

$$
p_{W}(y)=\sup _{w \in S_{W}}|w(y)| \quad(y \in Y) .
$$


Since $\nu(W)>0$ and $p_{W}(y) \leqq\|y\| \leqq \nu(W)^{-1} p_{W}(y)$ for each $y \in Y, p_{W}$ is a norm on $Y$ equivalent to the original norm and $\|w\|=p_{W}^{0}(w)$ for each $w \in W$, where $p_{W}^{0}$ denotes the dual norm in $\left(Y, p_{W}\right)^{\prime}$. Therefore, $\left(W, p_{W}^{0}\right)$ has characteristic equal to one in $\left(Y, p_{W}\right)^{\prime}$. Let $E^{1}$ and $F_{W}^{1}$ be the spaces obtained by replacing $Y$ with $\left(Y, p_{W}\right)$ and $W$ with $\left(W, p_{W}^{0}\right.$ ) in the definitions of $E$ and $F_{W}$, respectively. Since $E$ and $F_{W}$ are linearly homeomorphic to $E^{1}$ and $F_{W}^{1}$, respectively, it suffices, in the light of (i), to prove that every $\sigma\left(E^{1}, F_{W}^{1}\right)$-Cauchy sequence in $E^{1}$ is norm-bounded in $E^{1}$.

Let $\left\{T_{n}\right\}$ be a $\sigma\left(E^{1}, F_{W}^{1}\right)$-Cauchy sequence in $E^{1}$. Then $\left\{T_{n}\right\}$ is $\sigma\left(E^{1}, F_{W}^{1}\right)$-bounded, whence $\sup _{n \in \omega}\left|w\left(T_{n} x\right)\right|<+\infty$ for each $x \in X$, $w \in W$. Fix $x \in X$ and vary $w$ throughout $W$. Since the canonical embedding $\phi$ of $\left(Y, p_{W}\right)$ into $\left(W, p_{W}^{0}\right)^{\prime}$ is an isometry, the sequence of scalars $\left\{p_{W}\left(T_{n} x\right)\right\}$ is bounded for each $x \in X$ by the BanachSteinhaus theorem. Since $X$ is complete, a second application of the Banach-Steinhaus theorem implies that $\left\{T_{n}\right\}$ is norm-bounded in $E^{1}$. Thus, the first assertion of (ii) is proved.

The second assertions of (ii) and (iii) may be proved simultaneously after two remarks have been made. Let $\left\{y_{n}\right\}$ be a $\sigma(Y, W)$-Cauchy sequence in $Y$. Assuming the hypothesis of (ii) there exists $M_{0}>0$ such that $p_{W}\left(y_{n}\right) \leqq M_{0}, n \in \omega$, since $\left(W, p_{W}^{0}\right)$ has characteristic equal to one in $\left(Y, p_{W}\right)^{\prime}$ and is closed in $\left(Y, p_{W}\right)^{\prime}$. Therefore, $\left\|y_{n}\right\| \leqq \nu(W)^{-1} M_{0}$, $n \in \omega$. Assuming the hypothesis of (iii) the sequence $\left\{y_{n}\right\}$ is again norm-bounded by a result of Levin and Saxon [3]. Thus, in either case, there exists $M>0$ such that $\left\|y_{n}\right\| \leqq M, n \in \omega$. Choose $x_{0} \in X$ and $x^{\prime} \in X^{\prime}$ such that $\left\|x_{0}\right\|=M,\left\|x^{\prime}\right\|=1 /\left\|x_{0}\right\|$, and $x^{\prime}\left(x_{0}\right)=1$. If $Z$ is the null space of $x^{\prime}$ in $X$ and $\left[x_{0}\right]$ is the (closed) linear span of $x_{0}$ in $X$ then $X=Z \oplus\left[x_{0}\right]$, whence each $x \in X$ can be uniquely written as $x=z_{x}+\alpha_{x} x_{0}$ for some $z_{x} \in Z$ and scalar $\alpha_{x}$. Put $T_{n}=x^{\prime} \otimes y_{n}, n \in \omega$, and note that $\mu\left(T_{n}\right) \leqq 1$. Since $T_{n} x=\alpha_{x} y_{n}$, for each $\sum x_{i} \otimes w_{i} \in F_{W}$

$$
\begin{aligned}
\left|\left(\sum_{i} x_{i} \otimes w_{i}\right)\left(T_{n}\right)-\left(\sum_{i} x_{i} \otimes w_{i}\right)\left(T_{m}\right)\right| \\
\leqq \sum_{i}\left|\alpha_{x_{i}}\right|\left|w_{i}\left(y_{n}\right)-w_{i}\left(y_{m}\right)\right|,
\end{aligned}
$$

from which it follows that $\left\{T_{n}\right\}$ is $\sigma\left(E, F_{W}\right)$-Cauchy. By hypothesis, there exists $T \in E$ such that $\left\{T_{n}\right\}$ is $\sigma\left(E, F_{W}\right)$-convergent to $T$, and since $\left\{y_{n}\right\}$ converges to $T x_{0}$ in the $\sigma(Y, W)$-topology, the second parts of (ii) and (iii) are proved.

Finally, we prove the first part of (iii). Given any $\sigma\left(E, F_{W}\right)$-Cauchy sequence $\left\{T_{n}\right\}$ in $E$, we define the linear mapping $T$ as in the proof of 
(i). If $x \in X$ then $\left\{T_{n} x\right\}$ is $\sigma(Y, W)$-Cauchy and hence norm-bounded in $Y[3]$. By the Banach-Steinhaus theorem there exists $C>0$ such that $\mu\left(T_{n}\right) \leqq C, n \in \omega$, whence

$$
\begin{aligned}
\|T x\| & \leqq \nu(W)^{-1} \sup _{w \in S_{W}}|w(T x)| \\
& =\nu(W)^{-1} \sup _{w \in S_{W}} \lim _{n}\left|w\left(T_{n} x\right)\right| \\
& \leqq \nu(W)^{-1} C\|x\| .
\end{aligned}
$$

Hence, $T$ is continuous and, as before, $T$ is the $\sigma\left(E, F_{W}\right)$-limit of $\left\{T_{n}\right\}$.

Remark. Suppose $X$ and $Y$ are Banach spaces, and let $\gamma$ be the greatest cross norm. It is known [6] that $\left(X \otimes_{\gamma} Y\right)^{\prime}=\mathfrak{L}\left(X, Y^{\prime}\right)$ and thus that $\psi=\gamma$ in this setting. The topology $\sigma\left(\mathcal{L}\left(X, Y^{\prime}\right), X \otimes_{\psi} Y\right)$ is therefore the weak-star topology on $\mathscr{L}\left(X, Y^{\prime}\right)$, whence it follows that $\mathfrak{L}\left(X, Y^{\prime}\right)$ and its norm-closed unit ball are $\sigma\left(\mathscr{L}\left(X, Y^{\prime}\right), X \otimes_{\psi} Y\right)$ sequentially complete. This can also be proven using (i) and the first assertion of (ii) in the theorem, independent of the duality under the cross norm $\boldsymbol{\gamma}$.

CoRollary. Let $X^{\prime} \hat{\otimes}_{\lambda} Y$ be the $\lambda$-completion of $X^{\prime} \otimes_{\lambda} Y$ for a Banach space $Y$ and $\lambda^{0}$ the dual norm in $\left(X^{\prime} \hat{\otimes}_{\lambda} Y\right)^{\prime}$.

(i) For every weakly sequentially complete Banach space $Y$, the Banach space $l_{1} \hat{\otimes}_{\lambda} Y$ is sequentially complete in the $\sigma\left(l_{1} \hat{\otimes}_{\lambda} Y, c \otimes_{\lambda^{0}} Y^{\prime}\right)$ topology. Thus, in particular, $l_{1} \hat{\otimes}_{\lambda} l_{1}$ is $\sigma\left(l_{1} \hat{\otimes}_{\lambda} l_{1}, c \otimes_{\lambda^{0}} m\right)$-sequentially complete.

(ii) Let $J$ be the canonical embedding from $X$ into $X^{\prime \prime}$ and $X^{\prime \prime} \mid J X$ the quotient space of $X^{\prime \prime}$ with $J X$ equipped with the usual norm. For each Banach space $X$ such that $X^{\prime \prime} \mid J X$ is separable, the space $X^{\prime} \hat{\otimes}_{\lambda} l_{1}$ is $\sigma\left(X^{\prime} \hat{\otimes}_{\lambda} l_{1}, \quad X \otimes_{\lambda^{0} m}\right)$-sequentially complete. Thus, $X \hat{\otimes}_{\lambda} l_{1}$ is

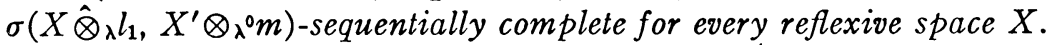

(iii) For every Banach space $X$ such that $X^{\prime \prime} \mid J X$ is separable, the

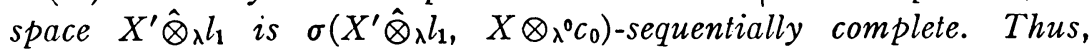
$X \hat{\otimes}_{\lambda} l_{1}$ is $\sigma\left(X \hat{\otimes}_{\lambda} l_{1}, X^{\prime} \otimes_{\lambda}{ }^{0} c_{0}\right)$-sequentially complete for every reflexive space $X$.

Proof. It is known $[2$, p. $515, \# 35]$ that if $Y$ is weakly sequentially complete then $\mathcal{L}(c, Y)$ consists entirely of compact mappings. Since $c^{\prime}$ is isometrically isomorphic to $l_{1}$ and $l_{1}$ has the approximation property [5, p. 108], $\mathfrak{L}(c, Y)=l_{1} \hat{\otimes}_{\lambda} Y$. Also, it is known [4] that if $X^{\prime \prime} \mid J X$ is separable, then $\mathscr{L}\left(X, l_{1}\right)=X^{\prime} \dot{\otimes}_{\lambda} l_{1}$. Both (i) and (ii) now follow from part (ii) of the theorem. Finally, (iii) follows directly from the remark since here also $\mathcal{L}\left(X, l_{1}\right)=X^{\prime} \hat{\otimes}{ }_{\lambda} l_{1}$. 
The relationship between $\nu(W)$ and $\nu\left(F_{W}\right)$ is precisely as one would expect.

Proposition. Let $X$ and $Y$ be normed linear spaces and $W$ a subspace of $Y^{\prime}$. Then $\nu(W)=\nu\left(F_{W}\right)$.

Proof. There exists $x^{\prime} \in X^{\prime}$ such that $\left\|x^{\prime}\right\|=1$. If $y \in Y$ and $f=\sum x_{i} \otimes w_{i} \in S_{F_{W}}$, then

$$
\left|\left(\sum x^{\prime}\left(x_{i}\right) w_{i}\right)(y)\right|=\left|f\left(x^{\prime} \otimes y\right)\right| \leqq\|y\|
$$

so that $\sum x^{\prime}\left(x_{i}\right) w_{i} \in S_{W}$. It follows that

Thus,

$$
\sup _{f \in S_{F_{W}}}\left|f\left(x^{\prime} \otimes y\right)\right| \leqq \sup _{w \in S_{W}}|w(y)| .
$$

$$
\begin{aligned}
\nu\left(F_{W}\right) & \leqq \inf _{\|y\|=1 ; \nu \in Y} \sup _{f \in S_{F_{W}}}\left|f\left(x^{\prime} \otimes y\right)\right| \\
& \leqq \inf _{\|y\|=1 ; y \in Y} \sup _{w \in S_{W}}|w(y)|=\nu(W) .
\end{aligned}
$$

To prove the reverse inequality, we define

$$
\mu_{W}(T)=\sup _{f \in S_{F_{W}}}|f(T)| \quad(T \in E)
$$

and note that

$$
\begin{aligned}
\mu_{W}(T) & \geqq \sup _{\|x\|=\|w\|=1}|x \otimes w(T)| \quad(x \in X, w \in W) \\
& =\sup _{\|x\|=1} \sup _{\|w\|=1}|w(T x)| \quad(x \in X, w \in W) \\
& \geqq v(W) \sup _{\|x\|=1}\|T x\| \quad(x \in X) \\
& =v(W) \mu(T) .
\end{aligned}
$$

Thus,

$$
\nu\left(F_{W}\right)=\inf _{\mu(T)=1} \mu_{W}(T) \geqq \nu(W) . \quad \triangle
$$

An extension of the theorem to the quasicomplete case exists and is not difficult to obtain. The author wishes to acknowledge this comment and other useful comments and suggestions made by the referee.

\section{References}

1. J. Dixmier, Sur un théorème de Banach, Duke Math. J. 15 (1948), 1057-1071. MR 10, 306. 
2. N. Dunford and J. T. Schwartz, Linear operators, I: General theory, Pure and Appl. Math., vol. 7, Interscience, New York, 1958. MR 22 \#8302.

3. M. Levin and S. Saxon, $A$ note on the inheritance properties of locally convex spaces by subspaces of countable codimension, Florida State University, Tallahassee, 1968 (unpublished).

4. R. D. McWilliams, On certain Banach spaces which are $w^{*}$-sequentially dense in their second duals, Duke Math. J. (to appear).

5. H. H. Schaefer, Topological vector spaces, 2nd ed., Macmillan, New York, 1967. MR 33 \#1689.

6. R. Schatten, A theory of cross spaces, Ann. of Math. Studies, no. 26, Princeton Univ. Press, Princeton, N. J., 1950. MR 12, 186.

Florida State University, Tallahassee, Florida 32306 